\title{
Muscle and skin improve by home-based FES and full- body in-bed gym
}

\author{
Carraro $U^{1,2,3 *}$, Albertin $G^{4}$, Gargiulo $P^{5}$, Ravara $B^{1,2}$, Piccione $F^{3}$, Zampieri $S^{1,6}$, Kern $\mathrm{H}^{6}$ and Amber Pond ${ }^{7}$ \\ ${ }^{1}$ Interdepartmental Research Center of Myology (CIR-Myo), Department of Biomedical Science, University of Padova, Italy \\ ${ }^{2} \mathrm{~A} \& \mathrm{C}$ M-C Foundation for Translational Myology, Padova, Italy \\ ${ }^{3}$ IRCCS Fondazione Ospedale San Camillo, Venezia-Lido, Italy \\ ${ }^{4}$ Section of Anatomy, Department of Neuroscience, University of Padova, Italy \\ ${ }^{5}$ Institute for Biomedical and Neural Engineering/Biomedical Technology Centre Reykjavik University and Landspitali, Reykjavik, Iceland \\ ${ }^{6}$ Physiko- und Rheumatherapie, St. Poelten, Austria \\ ${ }^{7}$ Anatomy Department, Southern Illinois University School of Medicine, Carbondale, Il, USA
}

\begin{abstract}
All progressive muscle contractile impairments, including advanced age-related muscle power decline, need permanent management. Most elderly persons, in particular octogenarians, spend small amounts of time in daily physical activity, resulting in a decline in body condition with more and more frequent hospitalizations and finally potentially forcing them to bed permanently. Further several neurological injuries, which are even more acutely debilitating than those problems related to aging, are responsible for early limitation of mobility. Inspired by the proven capability to recover skeletal muscle contractility and strength by home-based functional electrical stimulation (h-bFES) in both elderly and SCI patients, we suggest that the elderly and early aging patients participate in hbFES and add a 20 min daily routine of 12 easy and safe physical exercises, namely home-based Full-Body In-Bed Gym. Continued regularly, h-bFES and the Full-Body In-Bed Gym will help to maintain the independence of frail older people and may reduce the risks of serious consequences of accidental falls and pressure sore complications.
\end{abstract}

\section{Introduction}

There are about 700 named skeletal muscles in the human body, including 400 that only specialists care about. Better known are the roughly 300 skeletal muscles that are serious bone-movers, plus another 100 small, but important, muscles of the hands, feet, and face. The aim of this short report is to convince the elderly and people suffering with premature aging to combat muscle atrophy/sarcopenia/cachexia and thus maintain the health and good function of the majority of their body muscles, despite the fact that these tissues will inexorably decay decade after decade [1]. Skeletal muscle atrophy is the loss of muscle size and strength, which occurs with neural and skeletal muscle injuries, prolonged bed rest, space flight, normal aging, and diseases such as diabetes, sepsis and tumor cachexia, etc. If unabated, skeletal muscle atrophy can be extremely debilitating, increasing morbidity and mortality in affected people [2,3]. Because of advanced age and/ or associated/independent diseases, the elderly spends only a small amount of time in daily physical activity. The consequent loss of muscles (i.e., atrophy) further limits their activity and thus their independence, creating a situation that could eventually force them to bed and into hospitalization for long periods. Immobility-related muscle atrophy is associated with neuromuscular weakness, functional limitations, thromboembolism, and increasing medical costs [4-6]. All "progressive" muscle contractile impairments, muscle atrophy included, need permanent management. Unfortunately, pharmacological treatment of atrophy is not truly effective; thus a home-based physical exercise approach is extremely helpful in counteracting loss of muscle tissue. Awaiting the development of implantable devices for muscle stimulation, as effective as pacemakers for cardiac arrhythmias or cochlear implants for hearing loss, the education of sedentary patients as to the benefits of home physical exercises, whether these are volitional exercises and/or surface electrical stimulation during and after hospitalization, is a very cost-effective approach. Indeed, physical activity is an effective, low-cost alternative to hospitalization for rehabilitiation. Cardiovascular and ventilation rehabilitation of surgical patients are well established and a major component of these is intended to reverse muscle loss and weakness $[7,8]$. In previous studies, we have shown that denervated, atrophying muscles were rescued by 2-years of home-based functional electrical stimulation (h-bFES)

${ }^{\star}$ Correspondence to: Ugo Carraro, Department of Biomedical Science, Interdepartmental Research Centre of Myology, University of Padova, Padova, Italy; E-mail: ugo.carraro@unipd.it

Key words: muscle, skin, atrophy, h-bFES, Full-Body In-Bed Gym, elderly, borderline mobility impaired persons

Special Issue: Assisted Exercise

Ugo Carraro

Interdepartmental Center of Myology

University of Padova

Italy

Paolo Gargiulo

Inst. f. Biomed. and Neural

Engineering / Biomed Technology Centre

Reykjavik University \& Landspitali Reykjavik

Iceland

Received: May 10, 2018; Accepted: May 17, 2018; Published: May 24, 2018 
when a purpose developed electrical stimulator (now commercially available, "Stimulette den2x" of the Schuhfried Medizintechnik GmbH, Vienna, Austria) provided the needed high currents to large surface electrodes covering the Quadriceps muscles [9-15]. Interestingly, we recently demonstrated that the skin, when exposed to 2 years of h-bFES (to induce contractions of the atrophic Quadriceps muscles), shows an improvement in epidermis thickness [16]. Here we report that the electrical fields also produce clinically relevant recovery in atrophic Hamstrings muscles not in direct contact with the very large electrodes of the Vienna Protocol of h-bFES for denervated, atrophying muscles. What was considered a drawback (the lack of selectivity in muscle stimulation) has proven, indeed, to be an important clinical result.

\section{Materials and methods}

\section{Home-based Functional Electric Stimulation (h-bFES)}

Patients of the EU Program: RISE [Use of electrical stimulation to restore standing in paraplegics with long-term denervated degenerated muscles (QLG5-CT-2001-02191)] with complete Conus and Cauda Equina lesion were enrolled and gave appropriate informed consent. Using a custom-designed stimulator and large surface electrodes designed and implemented in Vienna (Austria), we stimulated denervated atrophic leg muscles according to the h-bFES strategy [14]. Measurements of knee torque were taken during electrical stimulation. Muscle biopsies were harvested before and after 2 years of h-bFES and analyzed by light and electron microscopy [14,21-23]. Muscle mass of the stimulated Quadriceps muscle were also determined using Quantitative Muscle Color Computed Tomography (QMC-CT) [1720]. QMC-CT is a highly sensitive quantitative imaging analysis of one group of anatomically defined skeletal muscles introduced by us to monitor skeletal muscles. QMC-CT is based on acquisition of high resolution CT scans and the use of special image processing tools allowing evaluation of soft tissues and skeletal muscle segmentation [17-20]. We developed QMC-CT as a by-product of the EU RISE project to complement follow-up in extreme cases of muscle degeneration, i.e., complete Conus and Cauda Equina syndrome, an SCI sequelae in which leg muscles are completely disconnected from the nervous system. QMC-CT uses CT numbers, i.e., Hounsfield Units (HU), for tissue characterization. In the process of assessing muscle quality, soft tissues were discriminated as follows: subcutaneous fat, intramuscular fat, low-density muscle, normal muscle, and fibrous-dense connective tissue (Figure 1). Pixels within the defined interval of $\mathrm{HU}$ values are selected and highlighted in colors (red for normal muscle tissue, yellow for intramuscular adipose tissue, green and blue for loose or fibrous connective tissue) while other tissues with $\mathrm{HU}$ values outside the threshold ranges remain black, included the extra-muscle adipose tissue [17-20].

\section{Full-Body In-Bed Gym}

Active persons, i.e., those able to make at least 25 consecutive push-ups in $3 \mathrm{~min}$, need the following exercises as a seasonal warm up to be able to perform very demanding physical activities. On the other hand, extreme sedentary people, after asking the advice of their family physician, may gradually start with five repetitions of each of the following suggested exercises. After the first or second week of training, they may add groups of five additional repetitions, up to 30 , every additional 1 or 2 weeks. If compliant, elderly people will progressively increase their muscle mass and strength even by reaching and maintaining only 15 or 20 daily repetitions. At the beginning, it will be safer to perform the routine at a very slow speed. When the
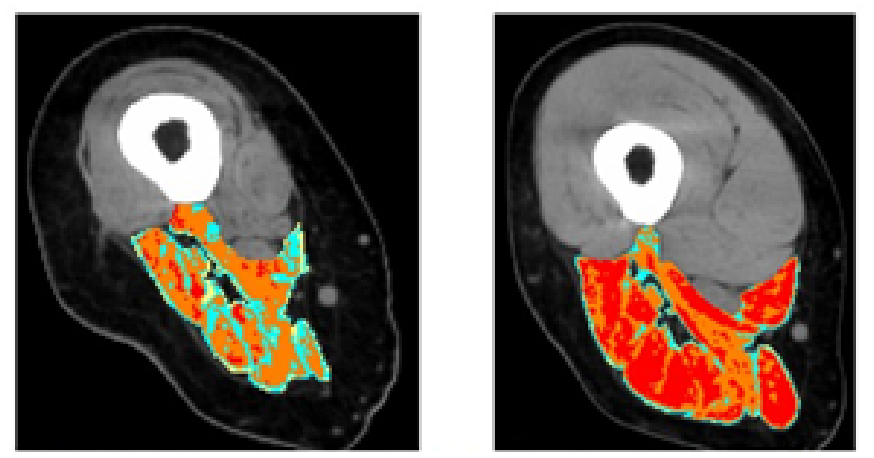

Right Leg Ham String muscles of single patient which started h-bFES 3 years after SCI. Left panel imaged at 3 year post SCI with no h-bFES treatment. Right panel imaged at 5 years post SCI with last teo years including h-bFES treatments

Figure 1. Muscle Color Computed Tomography of thigh muscles at $20 \mathrm{~cm}$ from femur head. Both the Quadriceps and the Hamstring muscles increased in size and tissue density (improved content of the red healthy muscle fibers) after two years of training using the Vienna protocol for h-bFES of permanently denervated human muscles. It is worth noting that, even starting at 3 years post SCI, h-bFES is able to recover the Hamstring muscles substantially

maximum number of repetitions of each exercise is reached, improved effects will be obtained by speeding up the exercises. The daily routine may last from 10 (in the beginning) to $30 \mathrm{~min}$ (for complete session in accustomed persons).

\section{Results}

\section{Home-based Functional Electric Stimulation (h-bFES)}

We reported that 20 out of 25 patients of the EU Program: RISE [Use of electrical stimulation to restore standing in paraplegics with long-term denervated degenerated muscles (QLG5-CT-2001-02191)] completed a 2-year h-bFES program, which resulted in: 1) a 35\% increase in cross-sectional area of the Quadriceps muscles ( $<<0.001)$; 2 ) a 75\% increase in mean diameter of Quadriceps muscle fibers $(\mathrm{p}<0.001)$; and 3) improvement of the ultrastructural organization of contractile material and of the $\mathrm{Ca}^{+}$-handling system [14]. Furthermore, a truly impressive $1187 \%$ increase in force output during electrical stimulation occurred ( $\mathrm{p}<0.001$ ) that was sufficient to allow $25 \%$ of the end-point subjects to perform FES-assisted stand-up exercises [14].

Though not expected, after the 2 years during which the 20 SCI subjects performed h-bFES 5 days per week by large electrodes covering the Quadriceps muscles, the CT cross sectional area of the Hamstring muscles also improved, increasing from $26.9+/-8.4(\mathrm{~cm} 2)$ to $30.7+/-9.8$ $\left(\mathrm{cm}^{2}\right)$, representing a significant $15 \%$ increase $(\mathrm{p} \leq 0.05)$ [14]. QMC-CT analyses confirm that h-bFES-induced muscle improvements (noted in CT of Quadriceps muscle) are present also in Hamstring muscles. Quadriceps and the Hamstring muscles increased in size and tissue density (improved content of healthy muscle fibers) after two years of training using the Vienna protocol for h-bFES for permanently denervated human muscles. It is worth noting that, even starting at 3 years post SCI, h-bFES is able to recover the Hamstring muscles substantially.

\section{Full-Body In-Bed Gym}

If sedentary persons (without major comorbidities, but with rest-related muscle weakness) challenge themselves, the full-body in-bed gym increases their muscle strength, fatigue resistance, and independence in daily life activities. 
For an educational video see at the link: http://www.bio.unipd.it/ bam/video/InterviewCarraro-tutorial.mp4

\section{Discussion}

Persons suffering with SCI must use wheelchairs to gain some mobility independence, and this results in them sitting on their Hamstring and Gluteal muscles for several hours each day. The prolonged seating contributes to severe atrophy of the muscles and edema of the legs, with increased risks of decubitus ulcers and deep thrombophlebitis. Of particular importance in SCI is whether the connection between the muscle and the nerve is preserved or the muscle is denervated due to complete peripheral nerve lesion. In the latter cases, the denervated muscle becomes unexcitable with typical commercial electrical stimulators and undergoes ultrastructural disorganization within a few months, while severe atrophy with nuclear clumping and fibro-fatty degeneration appears later on within 3 and 6 years $[2,3]$. Our work with h-bFES and our specific stimulator and protocols is important because it leads to muscle recovery, specifically in the worst case of complete, permanent lower motor neuron muscle denervation. Further, the improvement in Hamstring muscles contributes to the cushioning provided by all recovered muscle tissue and this is a major clinical benefit of the Vienna Protocol, validated by the EU Project RISE (Use of electrical stimulation to restore standing in paraplegics with long-term denervated degenerated muscles (QLG5CT-2001-02191) [14]. The h-bFES sustained increase in muscle mass is also important because of the increase in leg perfusion that recent analyses has demonstrated to be extended to the skin [16]. The improvement of muscle trophism will be even more clinically relevant if patients add h-bFES of Gluteal muscles to their training workout.

Anyhow, stimulators for neuromuscular electrical stimulation (ES) that are especially suited for elderly people requirements were designed and implemented in Vienna, Austria. As detailed in Kern et al., 2014 [24], older persons may be exposed to regular neuromuscular ES training. These constant voltage stimulation devices can be safely applied during home use. Starting two times a week, for a total amount of 24 training sessions $(3 \times 10$ minutes for each session) ES is safe and effective. Using this approach all subjects achieved a full knee extension. The outcome is a significant increase in muscle strength, associated with an increase of fast muscle fibers, which are the first to respond to ES and are well related to the power of skeletal muscle. ES significantly increased the size of fast type muscle fibers, and the number of Pax7- and NCAMpositive muscle satellite cells. Moreover, muscle biopsies did not present with signs of muscle damage and/or cellular inflammation [24-27].

\section{Conclusions}

In conclusion, we are doing our best to attract attention to our valuable results by publishing in high impact journals and book for experts [28-30]. Further dissemination is in the good hands of Editors of top medical journals and of Advisors of Granting Agencies. We are confident that they will share our desire to offer to people-in-need the chances to live a better life, as they deserve.

\section{Conflicts of interest}

The authors declare none conflict of interest regarding the publication of this paper.

\section{Funding statement}

The support of the European Regional Development FundCross Border Cooperation Program SLOVAKIA-AUSTRIA (Interreg- Iva) project 'Mobilität im Alter' MOBIL N_00033; Austrian Federal Ministry of Science and Research; Lud-wig Boltzmann Society (Vienna) is gratefully acknowledged. Supported also by institutional funds of the Research Center of Myology (CIR Myo) of the University of Padova, Italy, the IRCCS Fondazione Ospedale San Camillo, Venice, Italy and the A\&C M-C Foundation for Translational Myology, Padova, Italy.

\section{Acknowledgments}

UC and BR thanks for support A\&C M-C Foundation for Translational Myology, Padova, Italy. Research reported in this publication was supported in small part by the National Institute of Arthritis and Musculoskeletal and Skin Diseases of the National Institutes of Health under Award Number NIH NIAMS 1R03AR053706$01 \mathrm{~A} 2$ to ALP. The content is solely the responsibility of the authors and does not necessarily represent the official views of the National Institutes of Health.

\section{References}

1. Gava P, Kern H, Carraro U (2015) Age-associated power decline from running, jumping, and throwing male masters world records. Exp Aging Res 41: 115-135. [Crossref]

2. Kern H, Hofer C, Loefler S, Zampieri S, Gargiulo P, et al. (2017) Atrophy, ultrastructural disorders, severe atrophy and degeneration of denervated human muscle in $\mathrm{SCI}$ and Aging. Implications for their recovery by Functional Electrical Stimulation, updated 2017. Neurol Res 39:660-666. [Crossref]

3. Carraro U, Kern H, Gava P, Hofer C, Loefler S, et al. (2015) Biology of Muscle Atrophy and of its Recovery by FES in Aging and Mobility Impairments: Roots and By-Products. European Journal Translational Myology 25: 221-30. [Crossref]

4. Hopkins RO, Mitchell L, Thomsen GE, Schafer M, Link M, et al. (2016) Implementing a Mobility Program to Minimize Post-Intensive Care Syndrome. AACN Adv Crit Care 27: 187-203. [Crossref]

5. Camillo CA, Osadnik CR, van Remoortel H3, Burtin C4, Janssens W5, et al. (2016) Effect of "add-on" interventions on exercise training in individuals with COPD: a systematic review. ERJ Open Res 2. [Crossref]

6. Czyrny JJ, Kaplan RE, Wilding GE, Purdy CH, Hirsh J (2010) Electrical foot stimulation a potential new method of deep venous thrombosis prophylaxis. Vascular 18: 20-27. [Crossref]

7. Ades PA, Keteyian SJ, Wright JS, Hamm LF, Lui K, et al. (2017) Increasing Cardiac Rehabilitation Participation From 20\% to 70\%: A Road Map From the Million Hearts Cardiac Rehabilitation Collaborative. Mayo Clin Proc 92: 234-242. [Crossref]

8. Vorona S, Sabatini U, Al-Maqbali S, Bertoni M, Dres M, et al. (2018) Inspiratory Muscle Rehabilitation in Critically Ill Adults: A Systematic Review and Meta-Analysis. Ann Am Thorac Soc. [Crossref]

9. Kern H, Carraro U (2014) Home-Based Functional Electrical Stimulation for LongTerm Denervated Human Muscle: History, Basics, Results and Perspectives of the Vienna Rehabilitation Strategy. Eur J Transl Myol 24:3296. [Crossref]

10. Carraro U, Kern H (2016) Recovery from muscle weakness by exercise and FES lessons from Masters, active or sedentary seniors and SCI patients. Aging Clin Exp Res.

11. Sajer S (2017) Mobility disorders and pain, interrelations that need new research concepts and advanced clinical commitments. Eur J Transl Myol 27:7179. [Crossref]

12. Boncompagni S, Kern H. et al. (2007) Structural differentiation of skeletal muscle fibers in the absence of innervation in humans. Proc Natl Acad Sci U S A. 104: 1933919344. [Crossref]

13. Kern H, Carraro U, Adami N, Hofer C, Loefler S, et al. (2010) One year of home-based daily FES in complete lower motor neuron paraplegia: recovery of tetanic contractility drives the structural improvements of denervated muscle. Neurol Res. 32: 26-31. [Crossref]

14. Kern H, Carraro U, Adami N, Biral D, Hofer C, et al. (2010) Home-based functional electrical stimulation rescues permanently denervated muscles in paraplegic patients with complete lower motor neuron lesion. Neurorehabil Neural Repair 24: 709-721. [Crossref]

15. Available at: https://www.schuhfried.com/umbraco/Surface/AuthenticationSurface/ Login?return Url=\%2Fportal 
16. Albertin G, Hofer C, Zampieri S, Vogelauer M, Löfler S, et al. (2018) In complete SCI patients, long-term functional electrical stimulation of permanent denervated muscles increases epidermis thickness. Neurol Res. 40:277-282. [Crossref]

17. Gargiulo P, Reynisson PJ, Helgason B, Kern H, Mayr W (2011) Muscle, tendons, and bone: structural changes during denervation and FES treatment. Neurol Res vol. 33, pp. 750-758. [Crossref]

18. Carraro U, Edmunds KJ, Gargiulo P, et al. (2015) 3D False Color Computed Tomography for Diagnosis and Follow-Up of Permanent Denervated Human Muscles Submitted to Home-Based Functional Electrical Stimulation. Eur J Transl Myol 25:5133. [Crossref]

19. Edmunds KJ, Gíslason MK, Arnadottir ID, Marcante A, Piccione F, et al. (2016) Quantitative Computed Tomography and Image Analysis for Advanced Muscle Assessment. Eur J Transl Myol. 26:6015. [Crossref]

20. Edmunds K, Gíslason M, Sigurðsson S, Guðnason V, et al. (2018) Advanced quantitative methods in correlating sarcopenic muscle degeneration with lower extremity function biometrics and comorbidities. PLoS One 13: e0193241. [Crossref]

21. Kern H, Boncompagni S. et al. (2004) Long-term denervation in humans causes degeneration of both contractile and excitation- contraction coupling apparatus, wich is reversibile by functional electrical stimulation (FES). A role for myofiber regeneration? J Neuropathol Exp Neurol 63: 919-931.

22. Börjesson M, Onerup A, Lundqvist S, Dahlöf B (2016) Physical activity and exercise lower blood pressure in individuals with hypertension: narrative review of $27 \mathrm{RCTs} . \mathrm{Br}$ J Sports Med 50:356-361. [Crossref]

23. Krenn M, Haller M, Bijak M, Unger E, Hofer C, et al. (2011) Safe neuromuscular electrical stimulator designed for the elderly. Artif Organs 35: 253-256. [Crossref]
24. Kern H, Barberi L, Löfler S, Sbardella S, Burggraf S, et al. (2014) Electrical stimulation counteracts muscle decline in seniors. Front Aging Neurosci. 6: 189. [Crossref]

25. Zampieri S, Pietrangelo L, Loefler S, Fruhmann H, Vogelauer M, et al. (2015) Lifelong physical exercise delays age-associated skeletal muscle decline. J Gerontol A Biol Sci Med Sci. 70: 163-173. [Crossref]

26. Mosole S, Carraro U, Kern H, Loefler S, Fruhmann H, et al. (2014) Long-term highlevel exercise promotes muscle reinnervation with age. J Neuropathol Exp Neurol. 73 284-294. [Crossref]

27. Barber L, Scicchitano BM, Musaro A (2015) Molecular and cellular mechanisms of muscle aging and sarcopenia and effects of electrical stimulation in seniors. Eur $J$ Transl Myol. 25: 231-236. [Crossref]

28. Carraro U, Karma Gava K, Baba A, Piccione F, Marcante A (2016) Fighting muscle weakness in advanced aging by takehome strategies: Safe anti-aging full-body in-bed gym and functional electrical stimulation (FES) for mobility compromised elderly people. Biol Eng Med, 1:1-4.

29. Carraro U, Gava K, Musumeci A, Baba A, Piccione F, Marcante A (2018) Safe Antiaging Full-Body In-Bed Gym and FES for Lazy Persons: Home In-Bed Exercises for Fighting Muscle Weakness in Advanced Age. In: Rehabilitation Medicine for Elderly Patients, Masiero S, Carraro U, Eds., pag. 43-52. ISBN 978-3-319-57405-9 ISBN 978-3-319-57406-6 (eBook)

30. Carraro U, Gava K, Baba A, Marcante A, Piccione F. To contrast and reverse skeleta muscle atrophy by Full-Body In-Bed Gym, a mandatory life-style for older olds and borderline mobility impaired persons. Springer-Nature Book: Muscle Atrophy, Junjie Xiao, Ed., in press.

Copyright: $\odot 2018$ Carraro U. This is an open-access article distributed under the terms of the Creative Commons Attribution License, which permits unrestricted use, distribution, and reproduction in any medium, provided the original author and source are credited. 